Article

\title{
A Two-Step State Estimation Algorithm for Hybrid AC-DC Distribution Grids
}

\author{
Gaurav Kumar Roy *(D), Marco Pau (D), Ferdinanda Ponci and Antonello Monti
}

check for updates

Citation: Roy, G.K.; Pau, M.;

Ponci, F.; Monti, A. A Two-Step State Estimation Algorithm for Hybrid AC-DC Distribution Grids. Energies 2021, 14, 1967. https://doi.org/ $10.3390 /$ en14071967

Academic Editor: Luis M. Fernández-Ramírez

Received: 5 Mrach 2021

Accepted: 29 Mrach 2021

Published: 2 April 2021

Publisher's Note: MDPI stays neutral with regard to jurisdictional claims in published maps and institutional affiliations.

Copyright: (C) 2021 by the authors. Licensee MDPI, Basel, Switzerland. This article is an open access article distributed under the terms and conditions of the Creative Commons Attribution (CC BY) license (https:// creativecommons.org/licenses/by/ $4.0 /)$.
Institute for Automation of Complex Power Systems, RWTH Aachen University, 52062 Aachen, Germany; mpau@eonerc.rwth-aachen.de (M.P.); post_mod@eonerc.rwth-aachen.de (F.P.); amonti@eonerc.rwth-aachen.de (A.M.)

* Correspondence: groy@eonerc.rwth-aachen.de

\begin{abstract}
Direct Current (DC) grids are considered an attractive option for integrating high shares of renewable energy sources in the electrical distribution grid. Hence, in the future, Alternating Current (AC) and DC systems could be interconnected to form hybrid AC-DC distribution grids. This paper presents a two-step state estimation formulation for the monitoring of hybrid AC-DC grids. In the first step, state estimation is executed independently for the AC and DC areas of the distribution system. The second step refines the estimation results by exchanging boundary quantities at the AC-DC converters. To this purpose, the modulation index and phase angle control of the AC-DC converters are integrated into the second step of the proposed state estimation formulation. This allows providing additional inputs to the state estimation algorithm, which eventually leads to improve the accuracy of the state estimation results. Simulations on a sample AC-DC distribution grid are performed to highlight the benefits resulting from the integration of these converter control parameters for the estimation of both the AC and DC grid quantities.
\end{abstract}

Keywords: state estimation; grid monitoring; AC-DC grids; Multi-Terminal DC grids; voltage source converters

\section{Introduction}

The significant increase of renewable generation in the distribution system demands innovative solutions for the management of power grids. Multi-Terminal Direct Current (MTDC) grids are an interesting alternative to conventional network reinforcement. They offer an internal Direct Current (DC) link to connect distributed generation and allow higher system efficiencies and better utilization of the conducting materials with respect to Alternating Current (AC) grids [1,2]. MTDC grids are often discussed for their possible integration with AC transmission networks [3]. However, recent proposals also foresee the possible use of MTDC grids at distribution level. In this scenario, MTDC grids would be used to interconnect different Medium Voltage (MV) or Low Voltage (LV) AC grids, thus giving rise to hybrid AC-DC distribution grids. First implementations of AC-DC distribution grids can be already found in existing projects. In Reference [4], for example, a $\pm 5 \mathrm{kV}$ DC link is used for the connection to a $10 \mathrm{kV}$ AC grid using converters with 10 MVA rated power. In Reference [5], an existing $33 \mathrm{kV}$ double AC circuit is converted into a 30 MVA symmetrical monopole DC circuit operating at $\pm 27 \mathrm{kV}$. In these projects, MV AC and DC grids are interlinked via Voltage Source Converters (VSC), which are usually preferred over Line-Commutated Converters (LCC) for this type of applications [6]. The active and reactive power at the VSC is typically monitored and controlled by the Energy Management System (EMS) of the MTDC grid [7]. This control can be used to provide services to the AC grids, such as power peak shaving or support for contingencies resolution $[8,9]$.

One of the typical tools implemented by the EMS for grid monitoring is State Estimation (SE) [10]. The SE algorithms aim to reduce the error of the acquired measurements and 
provide accurate knowledge of the state of the network. The SE algorithms in the EMS use the measurements collected from the field to obtain an accurate picture of the operating conditions. Additional information, such as forecasts derived from historical data, can also be used to further improve the accuracy of the SE results [11]. In recent times, the increase of MTDC projects $[4,5,12]$ has led to the development of SE algorithms specific for hybrid AC-DC grids. In Reference [13], a distributed approach based on Lagrangian relaxation is proposed for the SE of hybrid AC/High Voltage DC (HVDC) grids. In Reference [14], a two-step procedure is presented where local estimations of the AC and DC grid states are refined via the exchange of the estimated active power flowing through the interfacing converters. In Reference [15], a dual decomposition approach is implemented for the coordination of a distributed SE algorithm. Reference [16] uses a three-stage approach to integrate mixed measurements and an Alternating Direction Multiplier Method (ADMM) for the distributed coordination. All the above proposals use conventional measurements to run the AC and DC system SE. The harmonization of the SE results between the AC and DC systems mainly relies on the exchange of information about the power flowing at the AC-DC converters.

However, additional information associated with the VSC operation and its control parameters can be essential to improve the monitoring of the hybrid AC-DC grid. The authors of Reference [17] presented a Weighted Least Square (WLS)-based SE algorithm, where the modulation index and phase angle of the VSC are used to couple the voltages at the AC and DC sides of a High Voltage DC (HVDC) link. Such work focuses on transmission grids with simple AC-DC interconnections. Thus, the possible effects of the proposed SE model in more complex MTDC grids is not thoroughly investigated. Similarly, the authors in Reference [18] consider the modulation index from the VSC as additional information for SE. This is introduced as a state variable in the AC-DC SE model. An equality constraint is also added to couple the $\mathrm{AC}$ and $\mathrm{DC}$ voltages at the two sides of the converters. The focus of Reference [18] is mainly on the beneficial effects that the converter control parameters bring in terms of redundancy for the detection of possible bad data. For this reason, a detailed analysis of the accuracy enhancements under standard conditions is missing. In Reference [19], a probabilistic SE approach for hybrid grids is presented. The proposed SE formulation integrates the DC grid model implicitly considering the knowledge of the modulation index. The goal of the paper is to deal with non-Gaussian uncertainties; hence, the specific benefits associated to the inclusion of the VSC control parameters is not analyzed. Recently, the work in Reference [20] presented a centralized WLS-based approach for hybrid HVDC/AC transmission networks. Additionally, in this case, the modulation indexes of the converters are used as coupling variables between AC and DC system. Provided results show that a centralized approach can provide better accuracy results than a distributed one. However, results of distributed SE approaches highly depend on how the coordination process is carried out. As an example, the work in Reference [21] demonstrates the key role of the uncertainty propagation for the final accuracy of the distributed SE formulation. In Reference [22], converter control parameters are also included into the SE framework. The converter details are exploited to integrate in the SE model the operation limits of the converter.

The authors of this paper have recently observed in Reference [23] that the internal converter measurements of current and voltage in the " $\mathrm{dq}$ " reference frame can improve the accuracy of the SE. However, VSC control parameters like modulation index and phase angle were not considered in the proposed formulation for AC-DC grid SE. In this paper, the two-step distributed SE procedure presented in Reference [14] is further developed to include the additional information from the VSC. In the first step of the SE algorithm, conventional measurements are processed, and the $A C$ and $D C$ states are separately estimated for the respective grids. In the second step, the AC and DC grid states are harmonized using both the information of the active power flowing through the converter and the modulation indexes extracted from the controller of the VSCs. The objective of this paper is presenting a two-step distributed SE algorithm for hybrid AC-DC grids that includes the 
VSC control parameters. Thus, the paper presents a method to perform the coordination process between $\mathrm{AC}$ and DC estimator. Moreover, this work aims at performing a detailed analysis of the impact that the inclusion of VSC parameters brings for the accuracy of the SE results. Table 1 shows the differences between the proposed SE approach and those already existing in the literature. The SE formulation here proposed has the typical benefits associated to distributed approaches, such as distributed computational burden, distributed communication requirements, and independent modeling of different systems. A more in-depth discussion about the strengths and weaknesses of centralized and distributed SE approaches is provided in Section 2. With respect to other distributed formulations, this proposal additionally provides the integration of the converter controller parameters, and it relies on a simpler (two-step) coordination process. Overall, the contributions of this paper are:

- $\quad$ presenting the integration of the VSC control parameters into a distributed two-step formulation of SE for hybrid AC-DC distribution grids;

- providing the details on how to perform the SE coordination process using the uncertainty propagation law to ensure the correct propagation of the uncertainties;

- proving the benefits in terms of SE accuracy resulting from the integration of the VSC control parameters in the SE algorithm.

Table 1. Overview State Estimation (SE) methods for hybrid Alternating Current-Direct Current (AC-DC) grids.

\begin{tabular}{ccccc}
\hline Reference & $\begin{array}{c}\text { SE } \\
\text { Method }\end{array}$ & $\begin{array}{c}\text { SE } \\
\text { Architecture }\end{array}$ & $\begin{array}{c}\text { Coordination } \\
\text { Process }\end{array}$ & $\begin{array}{c}\text { Converter } \\
\text { Parameters }\end{array}$ \\
\hline$[13]$ & WLS & distributed & iterative & no \\
{$[14]$} & WLS & distributed & two-step & no \\
{$[15]$} & WLS & distributed & iterative & no \\
{$[16]$} & WLS & distributed & iterative & yes \\
{$[17]$} & WLS & centralized & - & yes \\
{$[18]$} & LAV & centralized & - & yes \\
{$[19]$} & WLS & centralized & - & yes \\
{$[20]$} & WLS & centralized & - & yes \\
{$[22]$} & WLS & centralized & two-step & yes \\
This paper & WLS & distributed & & yes \\
\hline
\end{tabular}

The remainder of this paper is structured as follows. In Section 2, the general background on SE for hybrid AC-DC grids is given, and the centralized and distributed SE approaches are briefly introduced. Section 3 provides the details about the controller parameters that are used as additional measurements for the two-step SE. Section 4 then presents the methodology to include these converter parameters in the two-step SE algorithm. Section 5 analyzes the performance of the SE algorithm using a 14-bus AC-DC distribution grid as the benchmark, and it highlights the accuracy improvements possible, thanks to the integration of the converter parameters in the SE formulation. Finally, Section 6 concludes the paper.

\section{State Estimation for Hybrid AC-DC Grids}

\subsection{Weighted Least Squares Approach}

The SE in power systems is responsible for estimating the state of the grid by minimizing the influence of the errors unavoidably present in the measurements collected from the field. The formulation of the SE algorithms usually relies upon the measurement model shown in (1), which represents the mathematical relation between the measurements $\mathbf{z}$ collected from the grid and the state variables $\mathbf{x}$ chosen to represent the system:

$$
\mathbf{z}=\mathbf{h}(\mathbf{x})+\mathbf{e}
$$


In Equation (1), $\mathbf{z}$ is the vector of $M$ measurements, $\mathbf{x}$ is the state vector, $\mathbf{h}(\mathbf{x})$ is the vector of measurement functions linking the measured quantities to the state variables, and e represents the measurement error, which is associated to the limited accuracy of the measurement equipment. To ensure grid observability and measurement redundancy, the number of measurements in $\mathbf{z}$ must be larger than the number of state variables to be estimated in $\mathbf{x}$. The availability of an over-determined equation system is the prerequisite for the SE algorithm to filter out the measurement errors.

The measurement errors are typically considered as zero mean processes and assumed to be independent of each other. This assumption leads to the representation of the measurement error through a diagonal covariance matrix $\mathbf{R}$ having the following form:

$$
\mathbf{R}=\operatorname{Cov}(\mathbf{e})=E\left[\mathbf{e} \cdot \mathbf{e}_{T}\right]=\left[\begin{array}{ccc}
\sigma_{1}{ }^{2} & 0 & 0 \\
0 & \ddots & 0 \\
0 & 0 & \sigma_{M^{2}}
\end{array}\right]
$$

where $\sigma_{i}$ is the standard deviation of the $i$-th measurement.

Given this measurement model, the state of the grid can be estimated using different approaches. The most widely used approach is the Weighted Least Squares (WLS) method, which aims at minimizing the following cost function:

$$
J(x)=[\mathbf{z}-\mathbf{h}(\mathbf{x})]^{T} \mathbf{W}[\mathbf{z}-\mathbf{h}(\mathbf{x})] .
$$

In (3), the objective is to minimize the squared value of the so-called measurement residuals, namely the difference between each measurement and the corresponding quantity as obtained through the estimated state variables. In the WLS process, a weighting matrix $\mathbf{W}$, computed as the inverse of the covariance matrix $\mathbf{R}$, is also considered in order to provide more weight (and thus more importance) to more accurate measurements.

Due to the non-linearity of the measurement functions, the minimization of the WLS problem in (3) is usually obtained by iteratively solving (4):

$$
\Delta \mathbf{x}_{k}=\mathbf{x}_{k+1}-\mathbf{x}_{k}=\mathbf{G}_{k}^{-1} \mathbf{H}_{k}^{T} \mathbf{W}[\mathbf{z}-\mathbf{h}(\mathbf{x})],
$$

where $x_{k}$ is the estimated state vector at the $k$-th iteration, $\mathbf{H}_{k}$ is the Jacobian of the measurement functions $\mathbf{h}(\mathbf{x})$, and $\mathbf{G}_{k}=\mathbf{H}_{k}^{T} \mathbf{W} \mathbf{H}_{k}$ is the so-called Gain matrix. This iterative procedure is applied until the largest value (in absolute terms) of $\Delta \mathbf{x}_{k}$ falls below a predefined threshold.

With reference to the application of the WLS method for the SE of hybrid AC-DC grids, currently available proposals can be broadly classified in centralized single-step approaches, where the entire AC-DC grid is integrated into a unique model, and distributed approaches, where AC and DC grids are modeled and processed separately. The following subsections provide a short overview of the peculiarities of these two categories.

\subsection{Centralized SE for AC-DC Grids}

In a centralized single-step SE, both the AC and DC grid is integrated into the same model, and their electrical quantities are estimated within the same SE process. The proposals in References [17-20,22] are examples of these centralized solutions. In the following, the details of some of these approaches are presented to provide some insights on the pros and cons of centralized approaches.

The work in Reference [17] was the first proposal to include VSC control parameters into the SE model, referring explicitly to the integration of static synchronous compensators (STATCOM) and HVDC links within transmission system SE algorithms. To this purpose, the state vector of the AC grid is augmented by including the voltages at the DC side of the VSCs and their DC current. The resulting state vector is:

$$
\mathbf{x}^{T}=\left[V_{A C, 1}, \theta_{A C, 1}, \cdots, V_{A C, N_{A C}}, \theta_{A C, N_{A C}}, V_{D C, 1}, I_{D C, 1}, \cdots, V_{D C, N_{C}}, I_{D C, N_{C}}\right],
$$


where $V_{A C}$ and $V_{D C}$ are the voltage magnitudes at the AC and DC nodes, respectively, $\theta_{A C}$ is the phase angle of the AC nodes, and $I_{D C}$ is the current magnitude at the VSC. These quantities are needed for all the $N_{A C}$ buses of the AC grid and for all the $N_{c}$ buses corresponding to the DC side of a VSC.

The SE process uses conventional measurements on the AC grid (voltage, currents, powers) and possible measurements of the DC quantities at the VSC (DC voltage and DC current), as well as the Pulse-Width Modulated (PWM) modulation index (expressed as in (6)) and the phase angle control of the converter (namely the phase angle difference at the terminals of the coupling transformer of the VSC), as additional inputs coming from the VSC control to enhance the estimation results. The measurement functions associated to the VSC control quantities can be easily determined since they are clearly related to the state variables used in the augmented state vector.

$$
m=\frac{\sqrt{2} V_{A C}}{V_{D C}} .
$$

The SE formulation presented in Reference [18] refers more generally to hybrid AC-DC grids composed of MTDC grids. Even though a Least Absolute Value (LAV) estimator is used, the same concepts can be adopted to design also an analogous WLS estimator. In this case, the state vector is composed of the AC voltage variables (magnitude and phase angle) for all the $N_{A C}$ nodes of the AC grid, the DC voltage magnitudes for all the $N_{D C}$ nodes of the DC grid, and all the PWM modulation indexes for the $N_{c}$ converters in the system (note that the number of DC nodes and the number of VSCs can be generally different since the MTDC grid could have internal DC nodes not directly interfaced to the AC system). The resulting state vector in this case is:

$$
\mathbf{x}^{T}=\left[V_{A C, 1}, \theta_{A C, 1}, \cdots, V_{A C, N_{A C}}, \theta_{A C, N_{A C}}, V_{D C, 1}, \cdots, V_{D C, N_{D C}}, m_{1}, \cdots, m_{N_{c}}\right] .
$$

Similar to Reference [17], the SE algorithm accepts as input all the measurement quantities from the AC and DC grids, as well as the PWM modulation index extracted from the VSC control. The relationship between the AC and DC voltage at the VSC terminals is further reinforced by introducing Equation (6) as an equality constraint.

The main benefit of the centralized formulations is that the SE process for the entire AC-DC grid is carried out in a single step using a unique SE algorithm. For this reason, they also allow guaranteeing the best possible accuracy. On the other hand, having a centralized formulation requires integrating the complete AC-DC system within the same model and changing the SE software already in place within the distribution management systems. Moreover, this could lead to very large systems in which the MTDC grid is used to interconnect different $\mathrm{AC}$ distribution grids, resulting in heavy computational burden and high execution times for the state estimator.

\subsection{Distributed SE for AC-DC Grids}

In a distributed implementation of SE for hybrid AC-DC grids, the SE process can be decoupled between $\mathrm{AC}$ and $\mathrm{DC}$ grids so that each monitoring system is responsible for the estimation of its own quantities. The proposals in References [13-16] are all examples of distributed implementations of SE for hybrid AC-DC grids.

In the AC grid, the traditional choice of the state vector $\mathbf{x}_{A C}$ is given by the voltage magnitudes and phase angles at all the $N_{A C}$ nodes of the grid (see Equation (8)). The states $\mathbf{x}_{D C}$ of the DC system can be chosen as the set of the voltage magnitudes at all the $N_{D C}$ nodes of the DC grid (see Equation (9)). The state variables estimated in the two SE processes for the $\mathrm{AC}$ and the $\mathrm{DC}$ grid, respectively, thus, are the following:

$$
\begin{gathered}
\mathbf{x}_{A C}^{T}=\left[V_{A C, 1}, \theta_{A C, 1}, V_{A C, 2}, \theta_{A C, 2}, \cdots, V_{A C, N_{A C}}, \theta_{A C, N_{A C}}\right], \\
\mathbf{x}_{D C}^{T}=\left[V_{D C, 1}, V_{D C, 2}, V_{D C, 3}, \cdots, V_{D C, N_{D C}}\right] .
\end{gathered}
$$


The distributed framework allows decoupling also the collection of the measurement inputs between AC and DC monitoring system. Therefore, the AC grid measurements will feed the AC state estimator, whereas the measurement infrastructure on the DC network will provide the inputs for the DC state estimator. The key aspect for a distributed formulation is the harmonization of the results obtained on the AC and DC estimators. Different mathematical techniques can be adopted for such harmonization process, but in general, all of them rely upon the exchange of some boundary quantities that create a coupling constraint between the AC and DC grid. As an example, in Reference [14], the harmonization is performed in a second step by importing, in each estimator, the active power at the VSC as estimated by the other grid. The integration of this information is done by considering that the converter's active power at the $\mathrm{AC}\left(P_{c, A C}\right)$ and $\mathrm{DC}\left(P_{c, D C}\right)$ section differ only due to converter losses $P_{c, l o s s}$ :

$$
P_{c, A C}=P_{c, D C}+P_{c, l o s s}
$$

Equation (10) provides the relationship linking AC and DC active powers, thus allowing transferring these estimates from an estimator to the other. In this way, the local estimation can be refined by introducing the additional information estimated in the neighboring system in a second step.

The distributed SE formulation has some advantages compared to a centralized SE, such as faster computation time due to parallel execution of the SE for AC and DC grid and easier implementation of the SE algorithm. However, distributing the SE formulation usually leads to some degradation of the accuracy performance. To mitigate these degradations, complex coordination processes can be implemented. However, these would make the distributed formulation more complex and would bring more demanding communication requirements. More in general, it is also worth noting that, in future scenarios, the AC and DC grid could be independent entities managed by different distribution system operators. In this case, a distributed implementation would not be a choice but rather a requirement due to the independence of the monitoring systems. With a distributed framework, the SE process in both AC and DC grid can benefit from the information obtained from the neighboring grid without requiring an integrated implementation. The distributed formulations available at the moment usually consider the relationship in (10) as the primary coupling constraint between AC and DC grid. However, the VSC control parameters can provide further coupling constraints, as seen previously in Section 2.2. Hence, in the next section, the control parameters used in the VSC control scheme will be reviewed, with the goal to include them in a two-step distributed AC-DC SE algorithm.

\section{VSC Controller Parameter}

The VSC in the AC-DC system is used to exchange active and reactive power between the two grids. The control of the active and reactive power flow at the VSC is determined by the VSC controller, which varies according to the VSC topology. The converter topologies implemented in existing projects or widely used in the literature about VSCs are the twolevel converter, the three-level converter, and the Multi-Modular converter (MMC) [24-26]. The MMC converter is the preferred VSC topology for HVDC transmission links because of the involved high voltage levels and due to its better harmonic performance and smaller ripples in the output current towards the transmission grid [27]. Instead, the literature and projects on MV AC-DC distribution grids often use 3L-Neutral Point Clamped (NPC) converters because of their higher reliability with respect to MMCs [28]. Hence, in this paper, the 3L-NPC converter topology is assumed as VSC topology to describe the controller operation and derive the control parameters useful for SE purposes. However, it is worth noting that considerations and relationships similar to those finally obtained for the NPC converter also exist for other converter topologies, as shown in References [17,18]. Therefore, the relationships associated to the parameters to be integrated into the SE formulation (PWM modulation index and phase angle control) are not specific for the NPC converter, but they have a more general validity. 
The 3L-NPC converter controls the active and reactive power at its terminals using the PWM modulation scheme [29]. The VSC controller implements PWM modulation through an Outer Control Loop (OCL) and an Inner Current Loop (ICL) as depicted in Figure 1. The outer loop receives the operating points desired for the VSC and provides set-points to the inner loop. The inner loop calculates the reference parameters to generate PWM signals, which control the firing signals for the semiconductor switches in the VSC. This section briefly reviews the VSC controller operation and the VSC parameters which are used for the SE algorithm presented further in the paper.

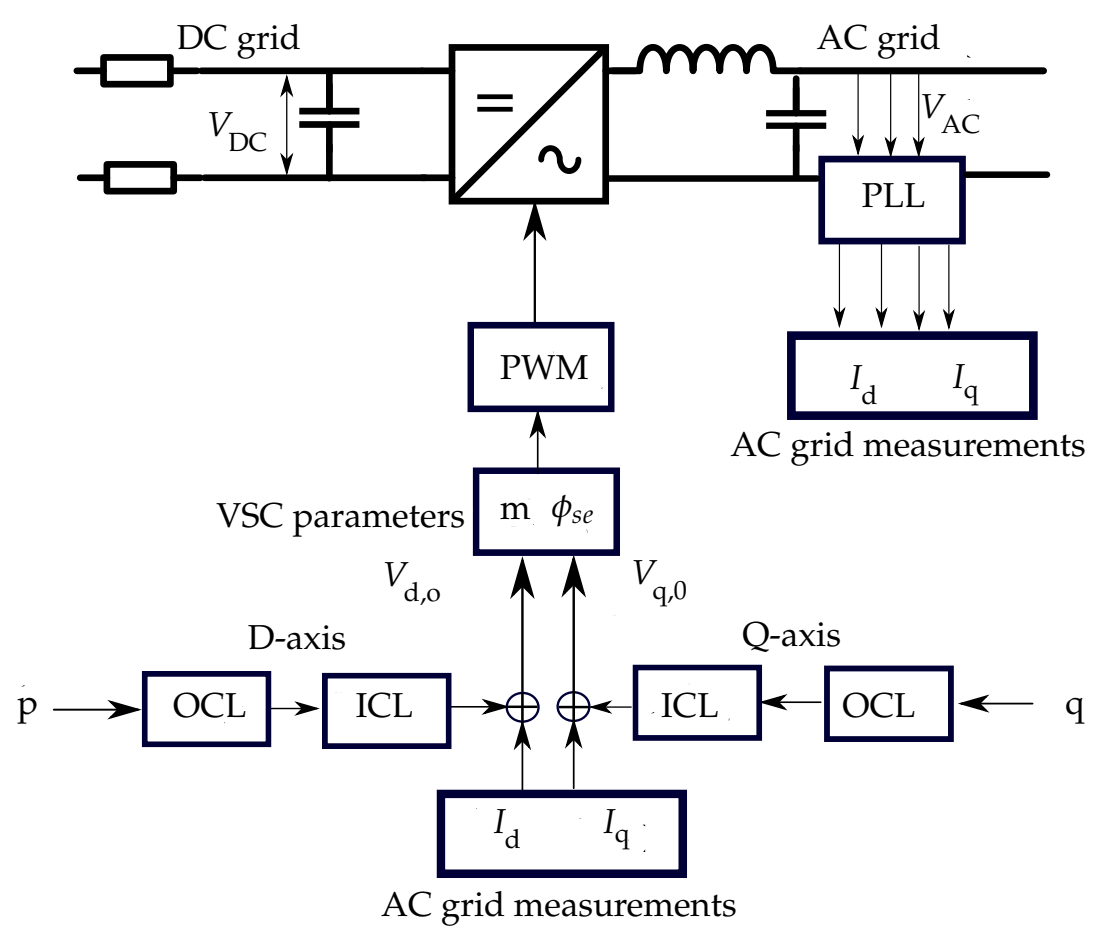

Figure 1. Schematic of Voltage Source Converter (VSC) control.

\subsection{VSC Control Structure}

The decoupled vector control or "dq" control is one of the most implemented control algorithms for 3L-NPC converter [26]. The VSC decoupled vector control is implemented in the dq-domain, where the set-points and measurements are processed in the outer loop and the inner current loop.

The VSC is assigned control actions to regulate active and reactive power in the MV AC-DC distribution grid. The VSC regulates active and reactive power flows to achieve control actions, such as regulating the voltage magnitude at the AC or DC terminals of the VSC or controlling the power flowing from one system to the other. The control actions is achieved by the VSC by controlling the active power and reactive power flow according to the active power set-point $p$ and the reactive power set-point $q$ given to the outer control loop (in the following, the internal converter set-points are labeled with the suffix, " 0 "). In the "dq" control, the three-phase AC currents and voltages measured at the point of common coupling are transformed into the dq-domain as $I_{d}$ and $I_{q}$ components for the current and $U_{d}$ and $U_{q}$ components for the voltage. The VSC is controlled to achieve the active power set-point $p$ by regulating the $d$-axis current component $i_{d, 0}$ represented by (11). Similarly, the reactive power $q$ in the VSC represented by (12) is regulated by controlling the q-axis component $i_{q, 0}$. The outer loop controls the active $p$ and reactive $q$ by generating the current components $i_{d, 0}$ and $i_{q, 0}$, which are current set-point sent to the inner loop [30]:

$$
p=\frac{3}{2}\left(U_{d} \cdot i_{d, 0}+U_{q} \cdot i_{q, 0}\right)=\frac{3}{2} U_{d} \cdot i_{d, 0},
$$




$$
q=\frac{3}{2}\left(U_{q} \cdot i_{d, 0}-U_{d} \cdot i_{q, 0}\right)=-\frac{3}{2} U_{d} \cdot i_{q, 0} .
$$

The inner loop in the VSC controller provides the current references $i_{d, 0}$ and $i_{q, 0}$ from the outer loop to the d-axis and q-axis Proportional Integral (PI) controllers in the inner loop and generates the control signals $u_{d, 0}$ and $u_{q, 0}$ [29]. The inner loop then calculates the voltage references $v_{d, 0}$ in the d-axis and $v_{q, 0}$ in the q-axis for the PWM generator in the VSC controller. The $v_{d, 0}$ is calculated from current measurements at dq-frame $I_{q}$ and voltage at d-axis $U_{d}$, represented in (13) and illustrated in Figure 1. In (13), $L$ is the inductance at the LC filter and $\omega$ is the angular frequency of the AC grid:

$$
v_{d, 0}=u_{d, 0}-L . \omega \cdot I_{q}+U_{d} .
$$

The voltage reference $v_{q, o}$ in q-axis for the PWM generator is calculated from the set-point $u_{q, 0}$ from the outer current loop and the dq-frame measurements $I_{d}$ and $U_{q}$, as shown in (14):

$$
v_{q, 0}=u_{q, 0}+L \cdot \omega \cdot I_{d}+U_{q} .
$$

The PWM strategy in the VSC generates a modulating signal, which controls semiconductor switches by turning them on and off to regulate the three phase voltage $V_{\mathrm{AC}}$ at the Point of Common Coupling (PCC) of the AC grid [29]. The PWM strategy, which is widely used for 3L-NPC VSC, is a carrier-based, pulse-width modulation strategy in which switching instants are determined by comparing the modulating signal with a carrier signal [26]. The carrier signal is a pre-determined high frequency periodic switching signal produced by the VSC, which is compared with the modulating signal. The modulating signal is controlled by the $v_{d, 0}$ and $v_{q, 0}$ as shown in (15). The magnitude $m$ and phase angle $\theta$ of the modulating signal is obtained by (15) and (16), respectively:

$$
\begin{gathered}
m=\frac{2 \sqrt{v_{d, o}^{2}+v_{q, o}^{2}}}{V_{D C}}=\frac{\sqrt{2} V_{A C}}{V_{D C}}, \\
\phi=\tan ^{-1} \frac{v_{d, 0}}{v_{q, o}} .
\end{gathered}
$$

The modulating index magnitude $m$ is a function that relates the Root Mean Square (RMS) of the AC voltage magnitude $V_{A C}$ and the DC voltage magnitude $V_{D C}$ at the terminals of the VSC. Hence, $m$, if known, can be used within SE to couple the voltages estimated at the AC and DC sides of the VSC. The voltage angle $\phi$ provided to the PWM generator indicates the phase angle difference between the PCC at the AC side of the grid and the AC terminals of the VSC, as shown in Figure 1. The $\phi$ control signal from the VSC, therefore, does not couple AC and DC quantities, but it can be still used as an input measurement of voltage angle for the $\mathrm{AC}$ grid $\mathrm{SE}$.

\subsection{VSC Parameters as Equivalent SE Measurements}

The presented two-step SE algorithm is used to monitor the quasi-static conditions of the AC-DC grid [5,12]. As a consequence, the VSC parameters for the two-step SE are modeled in this paper for steady state operating condition of the AC-DC grid, without considering the effects of fast transient disturbances [31]. The SE monitoring algorithm is assumed to execute at a lower bandwidth compared to the control loops of the VSC. Thus, in general, the time window for the SE should consider the higher bandwidth of the VSC parameters for the integration of the VSC parameters.

The VSC parameters $m$ and $\phi$ are calculated during a converter controller cycle time $T_{c}$. The converter controller cycle time $T_{c}$ is obtained from the maximum frequency $f_{\max }$ of the bandwidth by [32]:

$$
T_{c}=\frac{1}{2 \cdot f_{\max }}
$$


The SE, in comparison to the converter controller time $T_{c}$, is executed at a much lower frequency. For example, the MV AC-DC distribution grids in the Angle-DC project [5] are monitored with a time period of $0.5 \mathrm{~s}$. The SE is performed for AC and DC measurements collected over a time window of $T_{s e}$, whereas the controller parameters $m$ and $\phi$ are calculated with a time interval of $T_{\mathcal{c}}$. Hence, a SE time-window encompasses of $n_{s}$ samples of $m$ and $\phi$, as calculated below:

$$
n_{s}=\frac{T_{s e}}{T_{c}} .
$$

For the SE purposes, to have an information representative of the entire SE time window $T_{s e}$, the values averaged over time of PWM modulation index $\bar{m}$ and phase angle control $\bar{\phi}$ could, thus, be used, according to the following:

$$
\begin{gathered}
\bar{m}=\frac{\sum_{i=1}^{n_{s}} m_{i}}{n_{s}}, \\
\bar{\phi}=\frac{\sum_{i=1}^{n_{s}} \phi_{i}}{n_{s}} .
\end{gathered}
$$

When introducing the VSC control parameters as input for SE purposes, it is important not only to determine their value, but also the associated uncertainty to be considered. The main source of uncertainty for the actuation of the converter control parameters is related to the PWM generation process. In recent times, a PWM generation for state-of-the-art VSC FPGA-based controller has been assessed to have a standard deviation lower than 1\% [33]. This kind of information can be used to derive the uncertainties to be considered for the associated VSC control inputs in the WLS.

\section{Improved Two-Step State Estimation}

This section presents a distributed two-step SE algorithm that integrates the VSC control parameters $\bar{m}$ and $\bar{\phi}$ into the SE process. The proposed algorithm builds upon the SE logic presented in Reference [14]. In this paper, the VSC control parameters are considered as additional inputs to improve the accuracy performance of the estimator. The two steps of the algorithm consist of two different WLS estimation processes. In the first step, AC and DC state estimators run in parallel and independently using the measurements available in the $\mathrm{AC}$ and $\mathrm{DC}$ grid, respectively. As a result of this step, a first estimation of the states for the AC and DC grids is obtained. In the second step, the WLS estimation process is repeated, but with the inclusion of additional inputs coming from the estimation results of the other system. The data exchanged between the AC and DC systems to enable the second step are the active power through the VSC and the voltage magnitude at the VSC terminals. The flowchart in Figure 2 provides an overview of the steps performed by each estimator, together with the details of the needed data exchange. The details about the two steps and how the VSC control parameters are integrated into the proposed SE algorithm are given in the following subsections. 


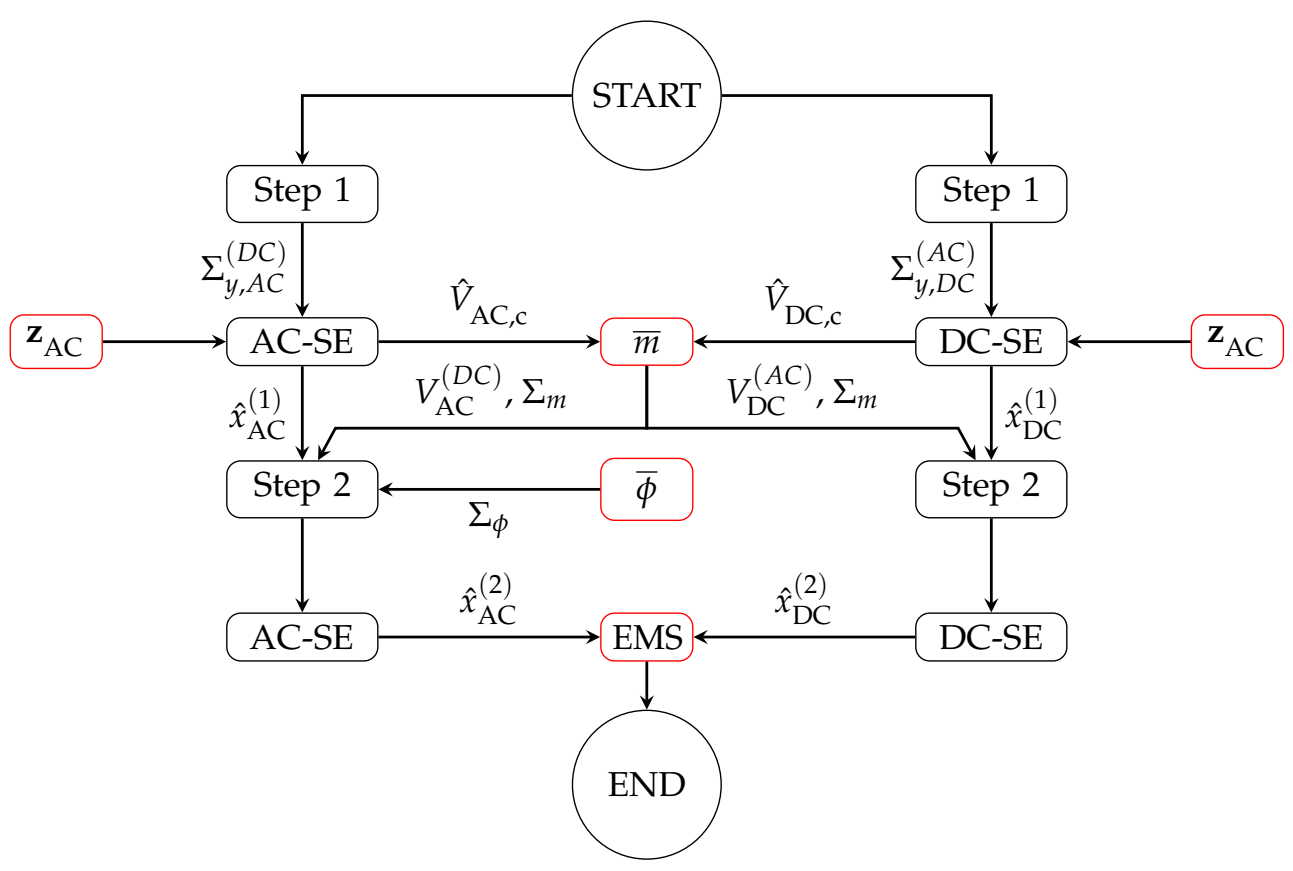

Figure 2. Algorithm for inclusion of VSC parameters in two-step SE.

\subsection{First-Step State Estimation}

The first step SE consists of a local SE run for both the AC and DC grid. Each grid estimator uses only the measurements available internally to its grid. The implemented AC state estimator accepts in input traditional AC measurements, such as voltage and current magnitude, active and reactive powers (either flowing in the branches or consumed/injected at the nodes), and last generation phasor measurements of voltage and currents coming from Phasor Measurement Units (PMU). Similarly, the DC state estimator uses only measurements available on the DC grid, such as voltage magnitude, current magnitude, and active powers. If available, both the estimators include measurements performed at the corresponding side (either AC or DC) of the VSCs, such as the voltage magnitude at the converter terminal and the power injected in the grid. The AC and DC estimators rely on the same state vectors $\mathbf{x}_{A C}$ and $\mathbf{x}_{D C}$ presented in Section 2.3. The WLS algorithm described in Section 2.1 is then used to obtain the first-step estimations $\hat{\mathbf{x}}_{A C}^{(1)}$ and $\hat{\mathbf{x}}_{D C}^{(1)}$. Besides, the covariance matrix $\Sigma_{\hat{\mathbf{x}}}$ of the estimated state vectors is computed via the inverse of the Gain matrix of the WLS. More information for the implementation of an AC distribution system state estimator able to process both traditional and PMU measurements can be found in either Reference [34,35]. Details about the mathematical expressions relevant for the design of the DC grid estimator are available in Reference [14].

The quantities to be exchanged between AC and DC estimators for the second step are: (i) the estimation of the voltage magnitude at the terminals of the VSCs; (ii) the active power injections at the VSC terminals. The needed voltages can be easily extracted from the WLS estimation results, as they are a subset of the state vector. The powers can be computed starting from the estimated variables, using the following expressions:

$$
\begin{gathered}
h_{P_{i}}(A C)=V_{i} \sum_{j \in \Gamma} g_{i j} V_{i}-V_{j}\left(g_{i j} \cos \theta_{i j}+b_{i j} \sin \theta_{i j}\right), \\
h_{P_{i}}(D C)=V_{i} \sum_{j \in \Gamma} g_{i j}\left(V_{i}-V_{j}\right),
\end{gathered}
$$

where $i$ is the generic node associated to the (AC or DC) VSC terminal, $\Gamma$ is the set of nodes adjacent to $i$, and $g_{i j}$ and $b_{i j}$ are the real and imaginary part of the series admittance of the line connecting nodes $i$ and $j$. 
The data exchange must include also the uncertainty information associated with the provided estimates. The last task within the first estimation step is, thus, to compute the covariance matrix $\Sigma_{y}$ of the data to be exchanged. This can be done by applying the law of propagation of the uncertainty, which results in the following:

$$
\Sigma_{y}=\left[\begin{array}{c}
H_{V} \\
H_{P}
\end{array}\right] \Sigma_{\hat{\mathbf{x}}}\left[\begin{array}{ll}
H_{V}^{T} & H_{P}^{T}
\end{array}\right],
$$

where $H_{V}$ is the sub-matrix pointing the voltage magnitude variables associated to the VSCs in the state vector, while $H_{P}$ is the matrix of partial derivatives of the previously presented functions $h_{P}$ with respect to the state vector variables.

It is worth noting that, as proven in Reference [21], the correct implementation of the second step requires considering the full covariance matrix $\Sigma_{y}$. This is essential since the correlation between different estimates can be high, especially for the case of the voltage estimates.

\subsection{Second-Step State Estimation}

In the second step, the AC grid acquires the voltage and power estimated by the DC estimator at the DC side of the VSCs (together with the associated covariance matrix) and vice versa. These quantities need then to be converted into equivalent quantities that can be used within the considered grid. Taking as reference the AC grid (the same procedure can be easily deducted also for the DC grid), the following converter constraints are used to convert the DC estimates into equivalent AC quantities:

$$
\begin{gathered}
V_{A C}=\frac{\bar{m} V_{D C}}{\sqrt{2}}, \\
P_{A C}=P_{D C}+P_{\text {losses }} .
\end{gathered}
$$

Equation (24) is the same coupling constraint also considered in References [17,18] to link the voltages at the two sides of the VSC. It shows that the knowledge of the PWM modulation index applied by the VSC controller is needed to move from the DC to the AC voltage (and vice versa). Equation (25) is the same coupling constraint initially considered in Reference [14]: it expresses the balance of the active power flow through the VSC.

In the second step, the WLS estimation process is repeated independently on the $\mathrm{AC}$ and DC grids. In this case, the two estimators use the information derived from the estimates provided by the other grid in addition to the starting measurements. Moreover, the additional VSC phase angle control $\bar{\phi}$ information is also appended to the set of inputs to the AC state estimator. Taking again the AC state estimator as an example, the following measurement vector $\mathbf{z}_{A C}^{(2)}$ is, thus, used in input to the second step WLS:

$$
\mathbf{z}_{A C}^{(2)}=\left[\begin{array}{lll}
\mathbf{z}_{A C}^{(1)} & \hat{\mathbf{y}}_{C, A C}^{(D C)} & \bar{\Phi}
\end{array}\right]^{T},
$$

where $\mathbf{z}_{A C}^{(1)}$ is the starting vector of measurements available in the AC grid (the same used during the first estimation step), $\hat{\mathbf{y}}_{C, A C}^{(D C)}$ is the vector of AC quantities at the VSCs derived from the corresponding DC estimates (it includes both voltage magnitudes and active powers), and $\bar{\Phi}$ is the vector of phase angle control inputs taken from the VSC controllers.

The overall covariance matrix of the second step measurement vector can be expressed as:

$$
\mathbf{R}_{A C}^{(2)}=\left[\begin{array}{ccc}
\mathbf{R}_{A C}^{(1)} & \mathbf{0} & \mathbf{0} \\
\mathbf{0} & \Sigma_{y, A C}^{(D C)} & \mathbf{0} \\
\mathbf{0} & \mathbf{0} & \Sigma_{\phi}
\end{array}\right]
$$


where $\mathbf{R}_{A C}^{(1)}, \Sigma_{y, A C}^{(D C)}$ and $\Sigma_{\phi}$ are the covariance submatrices associated to $\mathbf{z}_{A C}^{(1)}, \hat{\mathbf{y}}_{c, A C}^{(D C)}$, and $\bar{\Phi}$, respectively. In particular $\Sigma_{y, A C}^{(D C)}$ should be computed again by applying the law of propagation of the uncertainties to account for the uncertainties associated with the PWM modulation indexes. Using again the AC grid case as an example, this would translate into:

$$
\Sigma_{y, A C}^{(D C)}=\left[\begin{array}{ccc}
\mathbf{H}_{V_{A C}, m} & \mathbf{H}_{V_{A C}, V_{D C}} & \mathbf{0} \\
\mathbf{0} & \mathbf{0} & \mathbf{H}_{P_{A C}, P_{D C}}
\end{array}\right]\left[\begin{array}{cc}
\Sigma_{m} & \mathbf{0} \\
\mathbf{0} & \Sigma_{y_{D C}}
\end{array}\right]\left[\begin{array}{ccc}
\mathbf{H}_{V_{A C}, m} & \mathbf{H}_{V_{A C}, V_{D C}} & \mathbf{0} \\
\mathbf{0} & \mathbf{0} & \mathbf{H}_{P_{A C}, P_{D C}}
\end{array}\right]^{T},
$$

where the generic terms $H_{x, y}$ indicate the matrices of partial derivatives of the expression used to calculate the variable $x$ (Equation (24) or (25)) with respect to the variable $y$, while $\Sigma_{m}$ and $\Sigma_{y_{D C}}$ are the covariance matrices for the PWM modulation indexes and of the transmitted data from the DC grid as calculated via (23), respectively.

The inversion of the overall covariance matrix $\mathbf{R}_{A C}^{(2)}$ allows obtaining the weighting matrix to be used for the second step WLS process (the example here reported is for the AC grid, but the same procedure also holds for the DC grid). All the other terms of the WLS (namely measurement functions and Jacobian) can be computed as usual considering the specific electrical quantity represented in the measurement vector. The execution of the second step for both the AC and DC estimators eventually provides the final estimates for the $\mathrm{AC}$ and DC grids.

\section{Two-Step State Estimation Validation}

The proposed two-step SE algorithm has been tested using the 14-bus AC-DC grid shown in Figure 3, which consists of a three-node DC network connected to a nine-node AC system. The solution of a power flow problem calculated via the MATLAB tool MATACDC [36] is used to reproduce the operating conditions of the MV AC-DC distribution grid. The MATACDC power flow results are used as true reference values. Measurements are then generated from these reference values by adding random Gaussian noise to emulate the uncertainty of the measurements. The measurements are assumed to have Gaussian distributed error with a standard deviation equal to one-third of the measurement uncertainty (corresponding to a coverage factor equal to 3 ). The measurement error is, hence, created via random extraction from a Gaussian probability density function with zero mean and standard deviation equal to the above value.

The statistical evaluation of the SE accuracy performance has been performed via Monte Carlo simulations. In each of the scenario simulations 5000 Monte Carlo iterations have been performed to obtain statistically meaningful results. The Root Mean Square Error (RMSE) is chosen as indicator, as this is the most common index used to assess the accuracy performance of state estimators. The RMSE for the estimated states in the AC and DC nodes are calculated using (29):

$$
R M S E=\sqrt{\frac{1}{N_{M C}} \sum_{i=1}^{N_{M C}}\left(\hat{x}_{i}-x_{i}\right)^{2}},
$$

where $\hat{x}_{i}$ are the estimated values, $x_{i}$ the reference true values, and $N_{M C}$ the number of Monte Carlo iterations.

The two-step SE algorithm presented in the paper is tested for two scenarios. In the first scenario, the accuracy performance of the two-step SE is analyzed considering the input measurement set with and without the VSC parameters $m$ and $\phi$. The second scenario studies the effects on the SE algorithm of different accuracies for the modulation index $m$ and the phase angle $\phi$. 


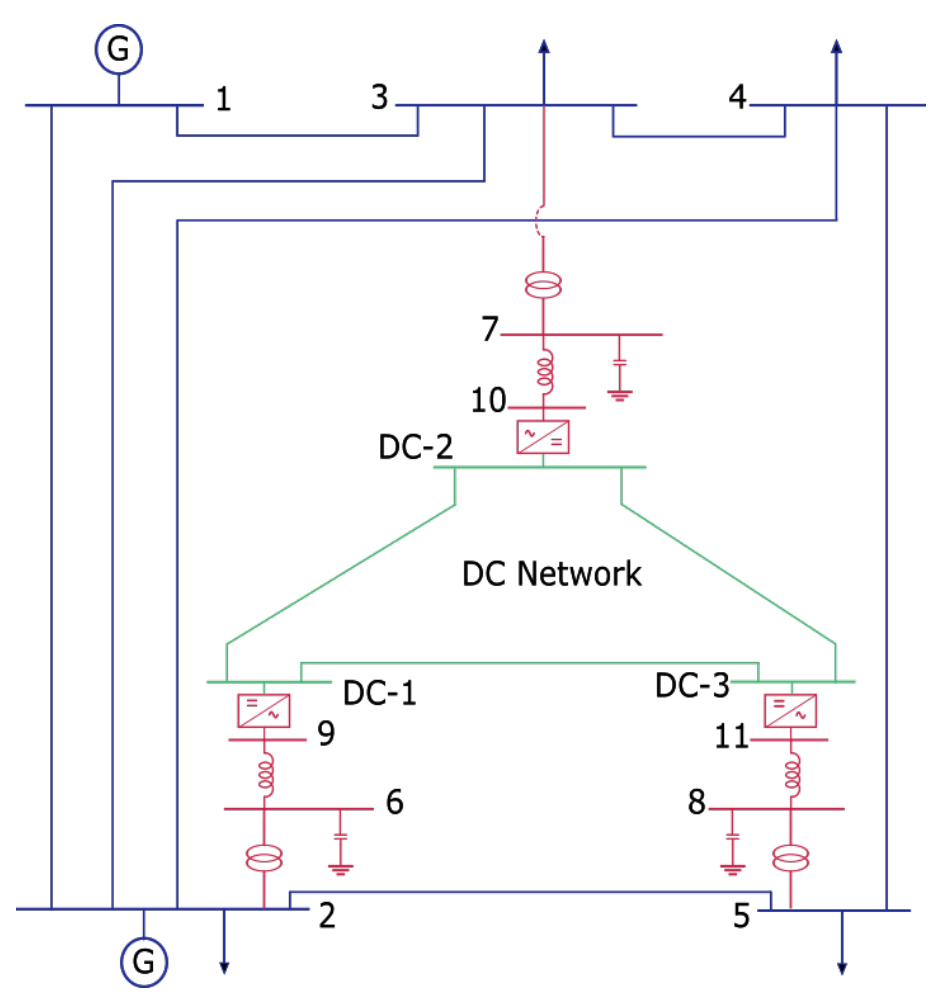

Figure 3. 14-Bus Medium Voltage (MV) distribution AC-DC grid.

\subsection{Scenario A: Impact of VSC Parameters}

In this scenario, two different measurement sets are used, which are termed measurement set $\mathrm{A}$ and measurement set $\mathrm{B}$. The measurement set $\mathrm{A}$ only considers conventional measurements from the AC and DC grid. As a result, the second step of the SE process in this case uses only the balance of the active power injections at the VSCs given by Equation (25). The measurement set B considers instead the availability of the VSC control parameters in addition to conventional measurements. The PWM modulation index $m$ and phase angle control $\phi$ of the converter are, hence, taken into account for the second step. Consequently, the second step uses both (24) and (25) to derive the additional inputs for the grid estimators and the phase angle control as further input for the AC estimator. The details of the measurement sets $\mathrm{A}$ and $\mathrm{B}$ are provided below:

- Set A: The AC grid measurements are: the voltage magnitude at nodes AC-4 and $\mathrm{AC}-5$; the active and reactive power for all the branches; the zero-power injection for the AC nodes AC-6, AC-7, and AC-8. The measurements considered for the DC grid are: the voltage magnitude at node DC-1; the current flow from bus DC-2 to bus DC-3; and the active power from node DC-3 to node DC-1. Two different uncertainty settings have been considered for the tests. The first one (case I) considers both AC and DC measurements with an uncertainty equal to $2 \%$ of the reading. In the second case (case II), DC measurements are considered with a better uncertainty, equal to $0.5 \%$ of the reading. The default measurement configuration used for the measurement set A is summarized in Table 2.

- Set B: The measurement set B consists of the same measurements used in set A along with the VSC parameters $m$ and $\phi$ for the three AC-DC converter nodes. For the tests in this scenario, the knowledge of the VSC parameters $m$ and $\phi$ is considered to have an uncertainty equal to $2 \%$. 
Table 2. Default measurement configuration.

\begin{tabular}{ccc}
\hline Measurement & AC Grid & DC Grid \\
\hline Voltage magnitude & $(4,5)$ & $(1)$ \\
Active power flow (from,to) & All branches & $(2-3)$ \\
Reactive power flow (from,to) & All branches & - \\
Current flow (from,to) & - & $(3-1)$ \\
Zero injections & $(6,7,8)$ & - \\
\hline
\end{tabular}

The performed tests prove that using the VSC converter control parameters allows improving the accuracy performance of the SE algorithm. Table 3 gives an example of the estimation results in a single SE shot against the true values. It is possible to observe that the results obtained with the measurement set $B$ are closer to the true value, which is an indication of the improvement brought by the inclusion of the VSC control parameters in the second SE step. However, for a statistically meaningful evaluation of the accuracy performance, the RMSE is a better indicator. Table 4 provides the RMSE and the standard deviation for the estimated states calculated at the second step of the SE process for both the measurement set A and set B. The RMSE and standard deviation values are almost identical, which is due to the fact that the WLS is an unbiased estimator. Table 4 also shows that clear improvements are obtained when introducing the VSC inputs, as done with the measurement set B. Figures 4 and 5 give a visual representation of these improvements for the AC and DC grid, respectively. For the AC grid, it can be observed that there is a slight improvement in the voltage magnitude estimation, with a reduction of the RMSE between $7 \%$ and $10 \%$. On the DC side, the obtained enhancements are much more evident. The reductions of the RMSE for this case are indeed up to $61 \%$. The differences in terms of obtained improvements between AC and DC grid are due to the different levels of uncertainty of the first step estimations. In fact, the first step voltage magnitude estimation in the AC grid is much more accurate than the one obtained by the DC estimator. As a result, during the second step, the voltage estimations coming from the DC estimator can bring only a small contribution for refining the accuracy of the AC voltage magnitude estimation. Vice versa, the accurate information imported from the AC grid is able to determine significant improvements on the estimation results of the DC voltage. As it can be observed in Figure 5, thanks to this contribution, the RMSE of the DC voltage estimation for measurement set $B$ is more than halved in comparison to measurement set A. Regardless of the different impact on the $\mathrm{AC}$ and DC grid, the obtained results prove that importing the voltage magnitude estimation from the neighboring system allows improving the accuracy of voltage magnitude estimations. The VSC modulation indexes are the inputs enabling the exchange of voltage magnitude estimations between $\mathrm{AC}$ and DC side. Therefore, their knowledge is essential to guarantee these improvements. The improvements for the voltage phase angle estimation depend on the use of the VSC phase angle control $\phi$. As shown in Figure 4, these improvements have a more local effect, as they are mostly concentrated on the buses associated with the VSCs.

Further tests have been performed to complement the results found in case I. As previously described, in case II, DC grid measurements are considered with a lower uncertainty equal to $0.5 \%$. Figure 6 shows the results of the voltage magnitude estimation in both the $\mathrm{AC}$ and $\mathrm{DC}$ grid for this test. On the $\mathrm{AC}$ grid, the obtained improvements are between $10 \%$ and $13 \%$, thus being slightly better than those achieved in case I. The reason for this is associated to the more accurate information received from the dc estimator, due to the lower uncertainty considered for the dc measurements. Vice versa, on the dc grid, the improvement obtained through the measurement set B is less significant than what was found in case I. The reduction of the RMSE is in fact around $25 \%$. The motivation behind this is associated to the better DC voltage estimation obtained at the first estimation step, which leaves less space for improvements. Even in this case, however, the beneficial contribution given by the imported AC voltages for the DC voltage estimation is still evident. 
Table 3. Scenario A: Estimated and true value comparison for 1 Monte-Carlo iteration.

\begin{tabular}{cccc}
\hline Voltage Node & True Value & Set A & Set B \\
\hline & & Estimation & Estimation \\
\hline AC-1 & 1.060 & 1.057 & 1.059 \\
AC-2 & 1.000 & 0.997 & 0.999 \\
AC-3 & 1.000 & 0.997 & 0.999 \\
AC-4 & 0.992 & 0.989 & 0.991 \\
AC-5 & 0.954 & 0.951 & 0.953 \\
AC-6 & 0.968 & 0.965 & 0.967 \\
AC-7 & 1.000 & 0.998 & 0.999 \\
AC-8 & 0.942 & 0.940 & 0.941 \\
AC-9 & 0.922 & 0.918 & 0.920 \\
AC-10 & 1.007 & 1.004 & 1.006 \\
AC-11 & 0.916 & 0.913 & 0.915 \\
DC-1 & 1.029 & 1.032 & 1.031 \\
DC-2 & 1.000 & 1.003 & 1.002 \\
DC-3 & 1.023 & 1.025 & 1.024 \\
\hline
\end{tabular}

Table 4. Root Mean Square Error (RMSE) and standard deviation comparison for set A and set B at second step SE.

\begin{tabular}{ccccccccc}
\hline Voltage Node & \multicolumn{9}{c}{ Set A } & \multicolumn{3}{c}{ Set B } \\
& \multicolumn{2}{c}{$\hat{\boldsymbol{V}}$} & \multicolumn{2}{c}{$\hat{\boldsymbol{\theta}}$} & & $\hat{\boldsymbol{V}}$ & & $\hat{\boldsymbol{\theta}}$ \\
\hline & RMSE & S.D & RMSE & S.D & RMSE & S.D & RMSE & S.D \\
\hline AC-1 & 0.134 & 0.134 & 0.00 & 00 & 0.124 & 0.125 & 0.00 & 00 \\
AC-2 & 0.147 & 0.147 & 0.0274 & 0.0274 & 0.137 & 0.137 & 0.0245 & 0.0247 \\
AC-3 & 0.146 & 0.146 & 0.0263 & 0.0263 & 0.137 & 0.135 & 0.0240 & 0.0241 \\
AC-4 & 0.148 & 0.146 & 0.0276 & 0.0276 & 0.138 & 0.137 & 0.0242 & 0.0241 \\
AC-5 & 0.157 & 0.159 & 0.0511 & 0.0515 & 0.147 & 0.149 & 0.0410 & 0.0412 \\
AC-6 & 0.159 & 0.154 & 0.0363 & 0.0363 & 0.147 & 0.149 & 0.0223 & 0.0226 \\
AC-7 & 0.150 & 0.148 & 0.0411 & 0.0412 & 0.138 & 0.134 & 0.0332 & 0.0322 \\
AC-8 & 0.165 & 0.161 & 0.0576 & 0.0575 & 0.151 & 0.157 & 0.0414 & 0.0411 \\
AC-9 & 0.184 & 0.182 & 0.0550 & 0.0554 & 0.166 & 0.167 & 0.0410 & 0.0414 \\
AC-10 & 0.149 & 0.146 & 0.0642 & 0.0643 & 0.135 & 0.130 & 0.0513 & 0.0514 \\
AC-11 & 0.171 & 0.173 & 0.0779 & 0.0778 & 0.157 & 0.159 & 0.0563 & 0.0573 \\
DC-1 & 0.482 & 0.482 & & & 0.187 & 0.188 & & \\
DC-2 & 0.510 & 0.510 & & & 0.197 & 0.194 & & \\
DC-3 & 0.489 & 0.489 & & & 0.190 & 0.192 & & \\
\hline
\end{tabular}
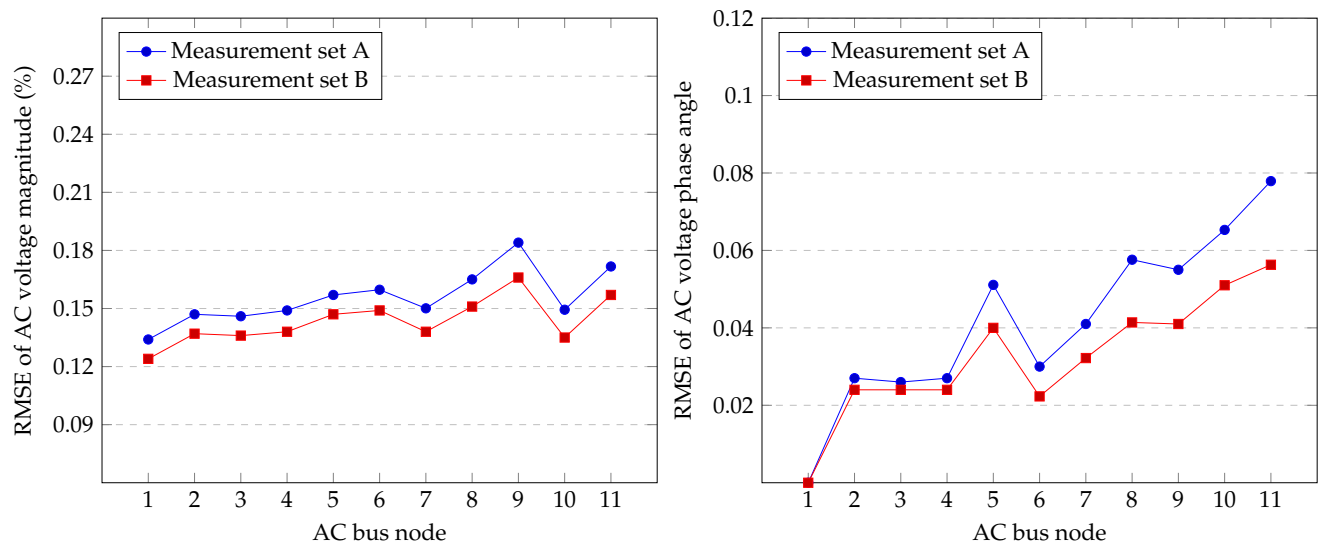

Figure 4. Scenario A: RMSE of estimated AC voltage for case I. 


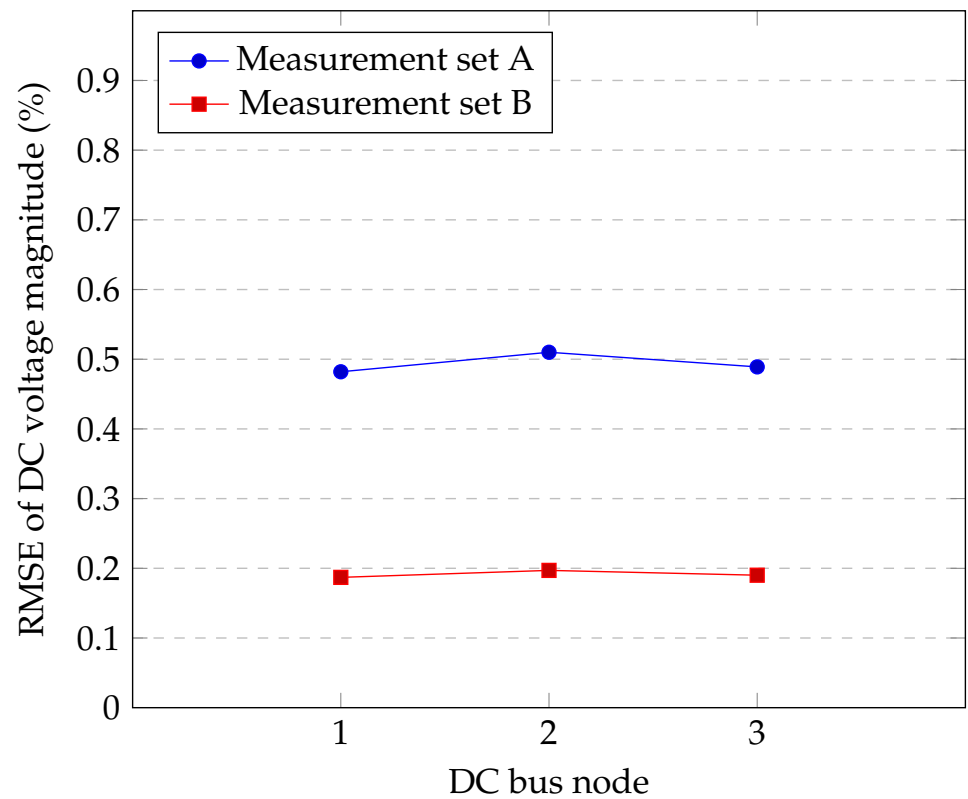

Figure 5. Scenario A: RMSE of estimated DC voltage for case I.
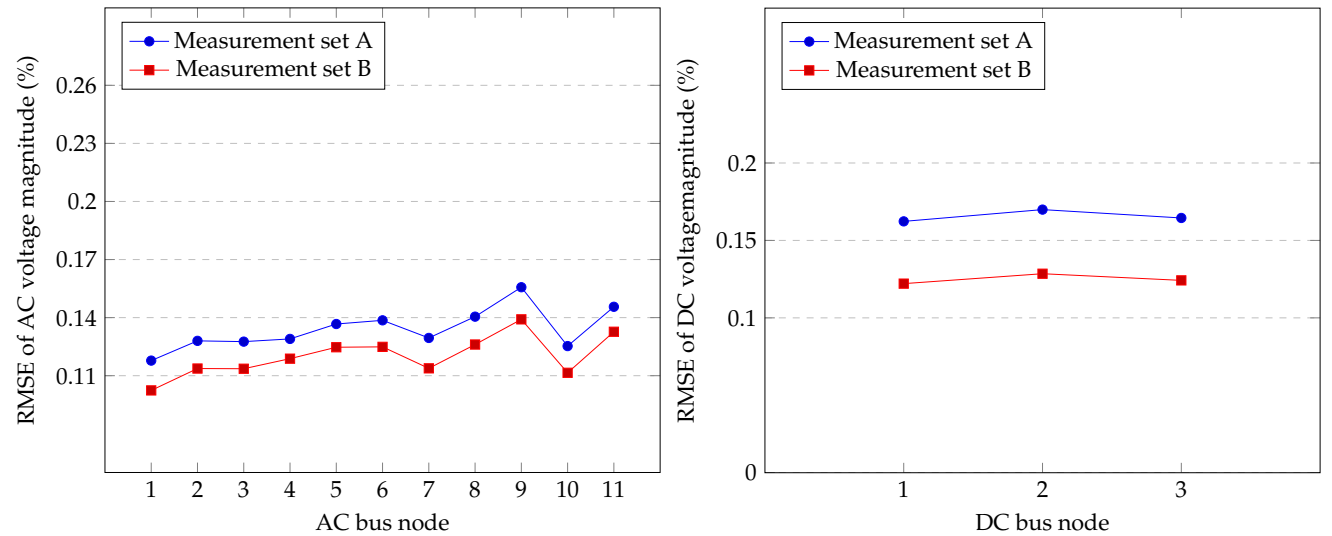

Figure 6. Scenario A: RMSE of estimated AC and DC voltage magnitudes for case II.

As a final result for this scenario, the statistical uncertainties obtained via the Monte Carlo simulation have been compared to the theoretical uncertainties extracted from the estimator through the inverse of the Gain matrix computed in the second step of the SE process. Figure 7 shows the estimated values of the voltage at the DC bus DC-1, together with the associated theoretical uncertainty obtained considering a coverage factor of 3 (the presented result is related to the first 50 Monte Carlo iterations of the case I test). The Figure clearly shows that the true value (in red) always lies within the estimation interval given by the computed uncertainty limits. Table 5 shows a summary of the statistical and theoretical uncertainties obtained in the same test. For the sake of brevity, only the DC grid results are reported, but the same considerations also hold for the AC grid estimation results. It is possible to observe that statistical and theoretical uncertainties always match, which indicates the correct implementation of the two-step SE algorithm and the proper management of the existing uncertainties via the uncertainty propagation process described in Section 4. 


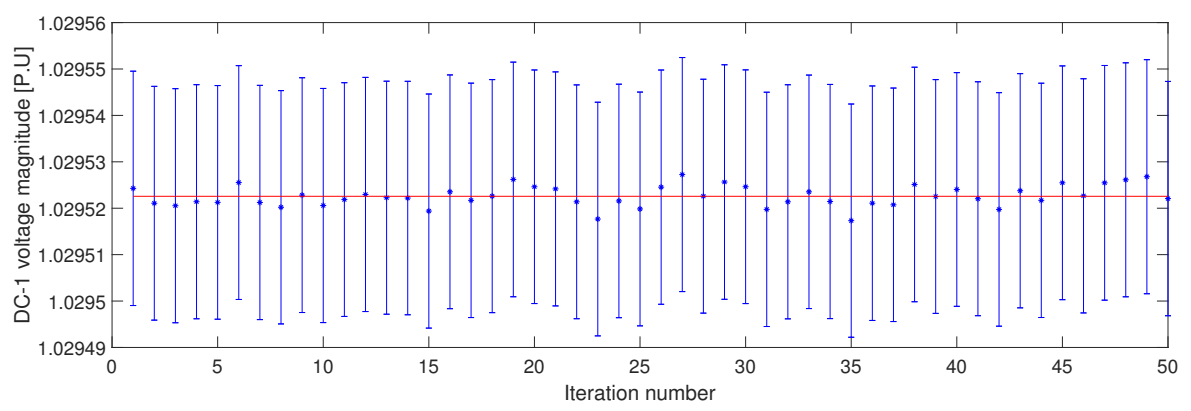

Figure 7. Statistical uncertainty plotted against theoretical uncertainty for DC-1.

Table 5. Statistical vs. theoretical uncertainty.

\begin{tabular}{|c|c|c|c|c|}
\hline \multirow[t]{2}{*}{ DC Voltage Node } & \multirow[t]{2}{*}{$\begin{array}{c}\text { Monte Carlo Uncertainty } \\
{[\%]}\end{array}$} & \multicolumn{3}{|c|}{$\begin{array}{c}\text { Theoretical Uncertainty } \\
{[\%]}\end{array}$} \\
\hline & & Average & Min. & Max \\
\hline DC-1 & 0.189 & 0.183 & 0.179 & 0.189 \\
\hline DC-2 & 0.195 & 0.193 & 0.191 & 0.199 \\
\hline DC-3 & 0.192 & 0.189 & 0.188 & 0.196 \\
\hline
\end{tabular}

\subsection{Scenario B: VSC Converter Parameter Uncertainty}

In this scenario, an additional set of tests has been carried out to investigate the impact of the uncertainties associated with the PWM modulation index. As shown in Section 4, the uncertainty of the PWM modulation index enters into the uncertainty propagation process, thus affecting the final uncertainty of the additional voltage magnitude inputs introduced in the second SE step. To analyze the impact of this source of uncertainty, tests have been performed by considering varying values of uncertainty for the modulation index. Figure 8 shows the impact of the modulation index uncertainty for the voltage estimation at the terminals of one of the AC-DC converters (namely for AC-9 and DC- 1 buses). It can be observed that lower levels of modulation index uncertainty lead to better estimation results. This is because the voltage magnitude estimations acquired from the other grid are less affected by the uncertainty of the modulation index. When reducing the uncertainty of the modulation index to values close to 0 , the RMSEs of the estimated voltages exhibit a saturation effect. This indicates that the estimation errors are approaching the lowest limit that is possible to achieve via the introduction of the voltages (with their uncertainties) from the neighboring grid. Tests with increasing uncertainties of the modulation index lead to RMSEs that tend asymptotically to the values found for the measurement set $\mathrm{A}$. This is because, for high modulation uncertainties, the uncertainty propagation process in (28) makes the voltages imported from the other grid very inaccurate. In these conditions, the contribution brought by the voltages taken from the neighboring grid is almost insignificant. 

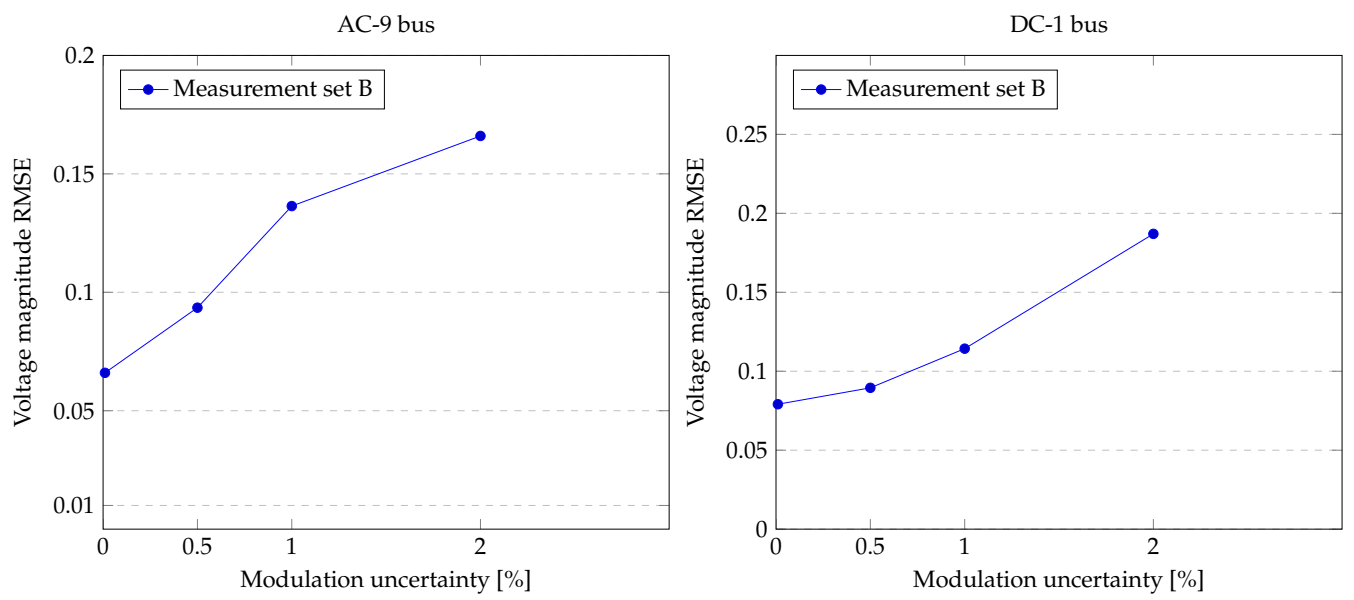

Figure 8. Scenario B: AC-9 bus and DC-1 bus voltage magnitude RMSE.

\section{Conclusions}

This paper presented a two-step SE algorithm for hybrid AC-DC grids that uses a distributed formulation to enable the independent monitoring of the AC and DC grid. The second step of the SE process allows importing in the AC and DC estimators the estimation of boundary quantities at the VSC, which is useful to improve the accuracy performance of the SE. In particular, this paper focused on the integration in the proposed distributed formulation of the VSC control parameters, namely the PWM modulation index $m$ and the phase angle control $\phi$, with the goal of understanding their impact on the SE results. Performed tests show that exploiting the VSC parameters allows improving the accuracy of the estimation results, both in the AC and in the DC grid. In some cases the achievable improvements can be also significant, as demonstrated in one of the considered scenarios, where the RMSE of the voltage magnitude at the DC buses resulted more than halved, thanks to the contribution brought by the inputs associated to the VSC control parameters. In general, the achievable accuracy improvements are strictly dependent on the measurement configuration and the measurement characteristics. This was confirmed through tests carried out with different levels of uncertainty for the DC measurements. Having a measurement infrastructure with better accuracy characteristics on the DC grid leaves less margins for improving the accuracy of the SE results (with the SE second step) on the same DC grid but brings higher improvements on the AC side. Tests also showed the potential impact associated to the uncertainty with which the modulation index is known. Low uncertainties lead to the possibility of achieving significant improvements both on the AC and the DC side, thus fully motivating the introduction of the VSC control parameters into the SE formulation. High uncertainties may lead instead to almost negligible improvements. In general, however, all the tests carried out in this paper demonstrated that the additional exchange of voltage magnitude estimations at the VSC terminals, which is unlocked by the introduction of the VSC modulation index, is highly beneficial for the enhancement of the SE accuracy performance. In the future, further extensions of this work may include the analysis of the bad data detection capabilities with the proposed distributed formulation, as well as a more detailed analysis of the uncertainty characteristics, for the converter control parameters. Testing and validation of the proposed SE solution in larger and more complex hybrid AC-DC grid scenarios, and in the real AC-DC grid being developed in the Campus of RWTH Aachen University, are additional activities planned to further strengthen the findings of this paper.

Author Contributions: Conceptualization, G.K.R. and M.P.; methodology, G.K.R. and M.P.; software, G.K.R.; validation, G.K.R. and M.P.; formal analysis, G.K.R.; resources, G.K.R.; data curation, G.K.R.; writing-original draft preparation, G.K.R. and M.P.; writing—review and editing, G.K.R., M.P. and F.P.; visualization, G.K.R. and M.P.; supervision, G.K.R., M.P., and F.P.; project administration, G.K.R.; 
funding acquisition, G.K.R., A.M., and F.P. All authors have read and agreed to the published version of the manuscript.

Funding: This research received no external funding.

Institutional Review Board Statement: Not applicable.

Informed Consent Statement: Not applicable.

Data Availability Statement: Not applicable.

Acknowledgments: The presented research and its results have been obtained in the project "DC-Sek: Control and automation of hybrid AC/DC distribution networks, in consideration of cyber-physical security aspects", funded by the German Federal Ministry of Education and Research (BMBF, funding code 03SF0594) under the roof of the Flexible Electrical Networks Research Campus.

Conflicts of Interest: The authors declare no conflict of interest.

$\begin{array}{ll}\text { Abbreviations } \\ \text { The following abbreviations are used in this manuscript } \\ \text { AC } & \text { Alternating Current } \\ \text { ADMM } & \text { Alternating Direction Multiplier Method } \\ \text { DC } & \text { Direct Current } \\ \text { EMS } & \text { Energy Management System } \\ \text { HVDC } & \text { High Voltage DC } \\ \text { ICL } & \text { Inner Current Loop } \\ \text { LAV } & \text { Least Absolute Value } \\ \text { LCC } & \text { Line-Commutated Converters } \\ \text { MV } & \text { Medium Voltage } \\ \text { MTDC } & \text { Multi-terminal DC } \\ \text { MMC } & \text { Multi-Modular converter } \\ \text { NPC } & \text { Neutral Point Clamped } \\ \text { OCL } & \text { Outer Current Loop } \\ \text { PCC } & \text { Point of Common Coupling } \\ \text { PI } & \text { Proportional Integral } \\ \text { PWM } & \text { Pulse Width Modulation } \\ \text { PMU } & \text { Phasor Measurement Unit } \\ \text { RMSE } & \text { Root Mean Square Error } \\ \text { SE } & \text { State Estimation } \\ \text { STATCOM } & \text { Static Synchronous Compensator } \\ \text { VSC } & \text { Voltage Source Converter } \\ \text { WLS } & \text { Weighted Least Square } \\ & \end{array}$

\section{References}

1. Stieneker, M.; De Doncker, R.W. Medium-voltage DC distribution grids in urban areas. In Proceedings of the 2016 IEEE 7th International Symposium on Power Electronics for Distributed Generation Systems (PEDG), Vancouver, BC, Canada, 27-30 June 2016; pp. 1-7.

2. Azaioud, H.; Claeys, R.; Knockaert, J.; Vandevelde, L.; Desmet, J. A Low-Voltage DC Backbone with Aggregated RES and BESS: Benefits Compared to a Traditional Low-Voltage AC System. Energies 2021, 14, 1420. [CrossRef]

3. Rodriguez, P.; Rouzbehi, K. Multi-terminal DC grids: Challenges and prospects. J. Mod. Power Syst. Clean Energy $2017,5,515-523$. [CrossRef]

4. Stieneker, M.; Butz, J.; Rabiee, S.; Stagge, H.; Doncker, R.W.D. Medium-Voltage DC Research Grid Aachen. In Proceedings of the International ETG Congress 2015; Die Energiewende-Blueprints for the New Energy Age, Bonn, Germany, 17-18 November 2015; pp. 1-7.

5. $\quad$ Bryans, R.; Bebbington, M.; Yu, J.; Smith, K.; Knott, J.; Moon, A. Real time control of a distribution connected MVDC link. In Proceedings of the 13th IET International Conference on AC and DC Power Transmission (ACDC 2017), Manchester, UK, 14-16 February 2017; pp. 1-6.

6. Beerten, J.; Van Hertem, D.; Belmans, R. VSC MTDC systems with a distributed DC voltage control-A power flow approach. In Proceedings of the 2011 IEEE Trondheim PowerTech, Trondheim, Norway, 19-23 June 2011; pp. 1-6. 
7. Beerten, J.; Cole, S.; Belmans, R. Generalized Steady-State VSC MTDC Model for Sequential AC/DC Power Flow Algorithms. IEEE Trans. Power Syst. 2012, 27, 821-829. [CrossRef]

8. Maza-Ortega, J.M.; Mauricio, J.M.; Barragán-Villarejo, M.; Demoulias, C.; Gomez-Exposito, A. Ancillary Services in Hybrid AC/DC Low Voltage Distribution Networks. Energies 2019, 12, 3591. [CrossRef]

9. Hwang, S.; Song, S.; Jang, G.; Yoon, M. An Operation Strategy of the Hybrid Multi-Terminal HVDC for Contingency. Energies 2019, 12, 2042. [CrossRef]

10. Abur, A.; Gomez-Expòsito, A. Power System State Estimation. Theory and Implementation; Marcel Dekker: New York, NY, USA, 2004.

11. Della Giustina, D.; Pau, M.; Pegoraro, P.A.; Ponci, F.; Sulis, S. Electrical distribution system state estimation: Measurement issues and challenges. IEEE Instrum. Meas. Mag. 2014, 17, 36-42. [CrossRef]

12. Korompili, A.; Sadu, A.; Ponci, F.; Monti, A. Flexible Electric Networks of the Future: Project on Control and Automation in MVDC grids. In Proceedings of the International ETG Congress 2015; Die Energiewende-Blueprints for the New Energy Age, Bonn, Germany, 17-18 November 2015; pp. 1-8.

13. Donde, V.; Feng, X.; Segerqvist, I.; Callavik, M. Distributed State Estimation of Hybrid AC/HVDC Grids by Network Decomposition. IEEE Trans. Smart Grid 2016, 5, 974-981. [CrossRef]

14. Pau, M.; Sadu, A.; Pillai, S.; Ponci, F.; Monti, A. A state estimation algorithm for hybrid AC/DC networks with multi-terminal DC grid. In Proceedings of the 2016 IEEE PES Innovative Smart Grid Technologies Conference Europe (ISGT-Europe), Ljubljana, Slovenia, 9-12 October 2016; pp. 1-6.

15. Xia, N.; Gooi, H.B.; Chen, S.; Hu, W. Decentralized State Estimation for Hybrid AC/DC Microgrids. IEEE Syst. J. 2018, 12, 434-443. [CrossRef]

16. Kong, X.; Yan, Z.; Guo, R.; Xu, X.; Fang, C. Three-Stage Distributed State Estimation for AC-DC Hybrid Distribution Network Under Mixed Measurement Environment. IEEE Access 2018, 6, 39027-39036. [CrossRef]

17. de la Villa Jaen, A.; Acha, E.; Gomez-Exposito, A. Voltage Source Converter Modeling for Power System State Estimation: STATCOM and VSC-HVDC. IEEE Trans. Power Syst. 2008, 23, 1552-1559. [CrossRef]

18. Mouco, A.; Abur, A. Robust L1 Estimators for Interconnected AC/DC Power Systems. In Proceedings of the 2019 IEEE Milan PowerTech, Milan, Italy, 23-27 June 2019; pp. 1-6.

19. Huang, Y.; Xu, Q.; Hu, C.; Sun, Y.; Lin, G. Probabilistic State Estimation Approach for AC/MTDC Distribution System Using Deep Belief Network With Non-Gaussian Uncertainties. IEEE Sens. J. 2019, 19, 9422-9430. [CrossRef]

20. Ayiad, M.; Leite, H.; Martins, H. State Estimation for Hybrid VSC Based HVDC/AC Transmission Networks. Energies 2020, 13, 4932. [CrossRef]

21. Sadu, A.; Pau, M.; Pillai, S.; Ponci, F.; Monti, A. Impact of uncertainty propagation on the design of state estimation for hybrid ac-dc grids. In Proceedings of the 2016 IEEE International Workshop on Applied Measurements for Power Systems (AMPS), Aachen, Germany, 28-30 September 2016; pp. 1-6.

22. Feng, W.; Shi, Q.; Cui, H.; Li, F.; Yuan, C.; Dai, R.; Liu, G. Using Lagrangian Relaxation to Include Operating Limits of VSC-MTDC System for State Estimation. In Proceedings of the 2020 IEEE Power Energy Society General Meeting (PESGM), Montreal, QC, Canada, 2-6 August 2020.

23. Roy, G.K.; Pau, M.; Sadu, A.; Ponci, F.; Monti, A. Inclusion of converter controller measurements into state estimation algorithm for hybrid ac-dc grid. In Proceedings of the 2019 IEEE Milan PowerTech, Milan, Italy, 23-27 June 2019; pp. 1-6.

24. Keshavarzi, M.D.; Ali, M.H. A Novel Bidirectional DC-DC Converter for Dynamic Performance Enhancement of Hybrid AC/DC Microgrid. Electronics 2020, 9, 1653. [CrossRef]

25. Rabiul Islam, M.; Mahfuz-Ur-Rahman, A.M.; Muttaqi, K.M.; Sutanto, D. State-of-the-Art of the Medium-Voltage Power Converter Technologies for Grid Integration of Solar Photovoltaic Power Plants. IEEE Trans. Energy Convers. 2019, 34, 372-384. [CrossRef]

26. Shah, R.; Sánchez, J.C.; Preece, R.; Barnes, M. Stability and control of mixed AC-DC systems with VSC-HVDC: A Review. IET Gener. Transm. Distrib. 2018, 12, 2207-2219. [CrossRef]

27. Debnath, S.; Qin, J.; Bahrani, B.; Saeedifard, M.; Barbosa, P. Operation, Control, and Applications of the Modular Multilevel Converter: A Review. IEEE Trans. Power Electron. 2015, 30, 37-53. [CrossRef]

28. Abeynayake, G.; Li, G.; Joseph, T.; Ming, W.; Liang, J.; Moon, A.; Smith, K.; Yu, J. Reliability Evaluation of Voltage Source Converters for MVDC Applications. In Proceedings of the 2019 IEEE Innovative Smart Grid Technologies-Asia (ISGT Asia), Chengdu, China, 21-24 May 2019; pp. 2566-2570.

29. Yazdani, A.; Iravani, R. A generalized state-space averaged model of the three-level NPC converter for systematic DC-voltagebalancer and current-controller design. IEEE Trans. Power Deliv. 2005, 20, 1105-1114. [CrossRef]

30. Yazdani, A.; Iravani, R. Dynamic model and control of the NPC-based back-to-back HVDC system. IEEE Trans. Power Deliv. 2006, 21, 414-424. [CrossRef]

31. Yazdani, A.; Iravani, R. A unified dynamic model and control for the voltage-sourced converter under unbalanced grid conditions IEEE Trans. Power Deliv. 2006, 21, 1620-1629. [CrossRef]

32. Sanchez-Ruiz, A.; Mazuela, M.; Alvarez, S.; Abad, G.; Baraia, I. Medium Voltage-High Power Converter Topologies Comparison Procedure, for a 6.6 kV Drive Application Using 4.5 kV IGBT Modules. IEEE Trans. Ind. Electron. 2012, 59, 1462-1476. [CrossRef]

33. Montano, F.; Ould-Bachir, T.; David, J.P. An Evaluation of a High-Level Synthesis Approach to the FPGA-Based Submicrosecond Real-Time Simulation of Power Converters. IEEE Trans. Ind. Electron. 2018, 65, 636-644. [CrossRef] 
34. Pau, M.; Pegoraro, P.A.; Sulis, S. Efficient Branch-Current-Based Distribution System State Estimation Including Synchronized Measurements. IEEE Trans. Instrum. Meas. 2013, 62, 2419-2429. [CrossRef]

35. Kong, X.; Chen, Y.; Xu, T.; Wang, C.; Yong, C.; Li, P.; Yu, L. A Hybrid State Estimator Based on SCADA and PMU Measurements for Medium Voltage Distribution System. Appl. Sci. 2018, 8, 1527. [CrossRef]

36. Beerten, J.; Belmans, R. MatACDC-An open source software tool for steady-state analysis and operation of HVDC grids. In Proceedings of the 11th IET International Conference on AC and DC Power Transmission, Stevanage, UK, 10-12 February 2015; pp. 1-9. 